\title{
Effect of Electrode Distance on Combustion Behavior of Fuel Droplet in Vertical DC Electric Field*
}

\author{
Osamu IMAMURA**, Bo CHEN**, Kiyotaka YAMASHITA***, \\ Shunsuke NISHIDA**, Mitsuhiro TSUE** and Michikata KONO**** \\ **The University of Tokyo \\ 7-3-1 Hongo, Bunkyo-ku, Tokyo 113-8656, Japan \\ E-mail: timamura@mail.ecc.u-tokyo.ac.jp \\ ${ }^{* * *}$ Colorado School of Mines \\ 1500 Illinois St., Golden, CO 80401, USA \\ ****National Institution for Academic Degrees and University Evaluation \\ 1-29-1 Gakuen-Nishimachi, Kodaira-shi, Tokyo 187-8587, Japan
}

\begin{abstract}
Flames are affected by external electric fields because they contain ions and electrons. The movement of ions and electrons affects the external electric field owing to their charge. In this context, this paper discusses the change in the electric field on the basis of experimental results obtained at different electrode distances. Employing one-dimensional (1D) steady-state analysis, we assume that if the electric field is changed spatially, the effect of the electric field on combustion behavior is aligned with $V^{2} / L^{3}$, where $V$ is the applied voltage between the electrodes and $L$ is the distance between the electrodes. Because a flame deforms to a cathode owing to electric body force in an electric field, the change in the flame shape of burning ethanol droplets observed in a vertical DC electric field and the electric current during combustion are measured as flame characteristics. The results reveal that applied electric voltage exists where the flame becomes vertically symmetrical to balance the buoyancy due to the electric body force. The relationship between $m$ and $n$ of $V^{m} / L^{n}$ for flame symmetry is around $n / m=1.5$. On the basis of these results, all experimental results for different electrode distances are rearranged with $\varepsilon V^{2} / L^{3}$, which is a representative electric body force, and it is proved that the use of parameter $\varepsilon V^{2} / L$ is effective. These results indicate that the change in the electric field due to the existence of a flame should be considered when examining the effect of an external electric field on combustion behavior.
\end{abstract}

Key words: Liquid Fuel, Flame, Diffusion Combustion, Combustion, Natural Convection, DC Electric Field, Droplet Combustion, Ethanol, Buoyancy, Electric Body Force, Ionic Wind

\section{Introduction}

Flames have electrical properties, as they contain many ions and electrons that are related to chemical reactions ${ }^{(1-4)}$. For example, when an external electric field is applied to a flame, the ions in the flame move through the electric field and collide with the molecules of neutral gas. The resultant electric body force on the flame is macroscopically apparent. This effect is known as "ionic wind." Because most effects on combustion that are induced by electric fields are related to this phenomenon, many studies have investigated and

*Received 15 Nov., 2010 (No. T1-09-0133) Japanese Original : Trans. Jpn. Soc. Mech. Eng., Vol.75, No.755, B (2009), pp.1532-1538 (Received 18 Feb., 2009) [DOI: 10.1299/jee.6.210]

Copyright $(\subset 2011$ by JSME 
reported on the aforementioned effect ${ }^{(1-4)}$. The movement of ions and electrons in the flame results in the generation of an electric field owing to their electric charges and affects the external electric field. Although these kinds of interactions between the flame and the electric field have been discussed ${ }^{(2)}$, some recent reports have summarized their results without considering the change in the external electric field. This has occurred for a variety of reasons; for example, it is difficult to estimate the electric charge density and to measure the electric field itself. We believe that another reason is that these experiments were conducted specifically for a unique experimental apparatus. In general, the experimental results are described using the value of the applied voltages $V$ divided by the electrode distance $L$ (hereinafter called the equivalent strength of the electric field and expressed as $\left.E_{e q}=V / L\right)$, under the assumption that the electric field between the electrodes is uniform. Even if the electric field changes and the effect of the electric field on the flame is described by $V^{m} / L^{n}(m \neq n)$, the difference between $V / L$ and $V^{m} / L^{n}(m \neq n)$ is negligible when $L$ is constant for the unique apparatus. This is why the effect of changes in the electric field is not explicitly discussed. Namely, if we compare the results obtained by the apparatus at different electrode distances, the change in the electric field should be explicitly considered.

In this context, the main purposes of this study are the investigation of combustion experiments at various electrode distances and the discussion of the distribution of the electric field around the flame through a comparison between several experimental results at various electrode distances. Droplet combustion experiments were performed in a vertical direct current (DC) electric field, in accordance with our previous paper ${ }^{(5)}$. In the previous paper, we demonstrated the possibility of controlling the natural buoyancy around burning a droplet using a vertical electric field. Although an attempt to balance the natural buoyancy using an electric field was made both experimentally and numerically by Papac et al. and others $^{(6-8)}$, the effect of the electrode distance was not mentioned and the change in the electric field was not clarified. In addition, although some research has discussed the effect of a change in the electric field around a flame, no expressions have clearly included the electrode distance effect. Against this background, in this paper, a one-dimensional (1D) steady-state analysis is described that includes the change in the electric field; the experimental results are rearranged based on the analysis. As a result, the experimental results and the analysis are in quite good agreement. The details of the 1D model and the experiment are reported herein.

\section{Experimental Apparatus and Method}

Figure 1 shows a schematic of the experimental apparatus, which is similar to that in the previous study ${ }^{(5)}$. The fuel droplet is supported by a silica fiber that is fixed horizontally. The fiber is $125 \mu \mathrm{m}$ in diameter, and the droplet diameter before combustion is around 1 $\mathrm{mm}$. The fiber is nonconductive and the electrons and ions in the flame are not able to escape through the support fiber. Parallel-plate electrodes are arranged above and below the droplet, and electric fields can be formed by applying electric voltage between them. The electrode is made of wire netting so as not to disturb the flow field around the droplet. The electrodes are square-shaped and are $50 \mathrm{~mm}$ on one side; the distance between them, $L$, ranges from 30 to $90 \mathrm{~mm}$. The droplet is always at the center position between the electrodes. An electric resistor of $1 \mathrm{M} \Omega$ is inserted in the electric circuit to measure the electric current during combustion. The applied electric voltage between the electrodes, $V$, ranges from 0 to $10 \mathrm{kV}$; the discharge was not observed in the applied voltage ranges. Ethanol is utilized as the fuel in this study, because it eliminates the complex effect of soot particles. The ambient fluid is air at room temperature and atmospheric pressure. The fuel droplet is ignited using a torch after the application of electric voltage between the electrodes. The flame shape is observed using a charge-coupled device (CCD) camera and 
the electric current is measured using a data logger.

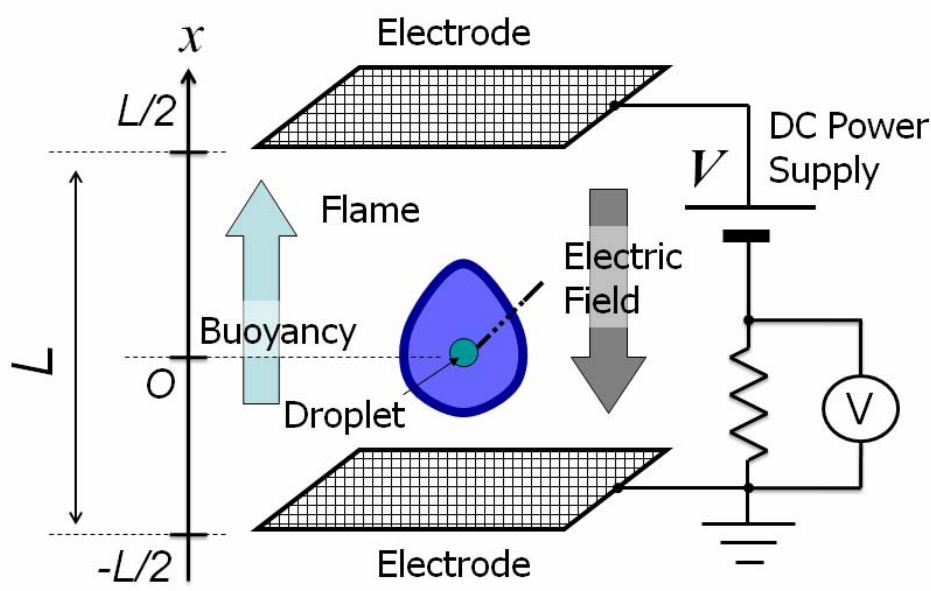

Fig. 1 Schematic of experimental apparatus

Table 1 Positive or negative signs of parameters

\begin{tabular}{|c|c|c|c|c|c|}
\hline Subscripts & $x$ & $j$ & $\mu$ & $e$ & $E$ \\
\hline $\mathrm{C}$ & - & - & + & + & + \\
\hline $\mathrm{A}$ & + & - & - & - & + \\
\hline
\end{tabular}

\section{Model Adopted in this Study}

\subsection{Introduction of electric body force}

As mentioned in the introduction, the movement of ions and electrons in a flame affects the external electric field. In this section, this effect is discussed with the help of a simple 1D steady-state model. The $x$-axis is defined to be in the upward direction and the origin is set at the center of the flame, as illustrated in Fig. 1. It is assumed that the carriers of electric charge, which are ions and electrons, appear only at $x=0$ and disappear only on the electrodes $(x= \pm L / 2)$. The unipolar model is applied, in which only positive ions exist in the region $x<0$ as electric charge carriers. The electric current density $j$ (which is negative in this definition) is constant between the electrodes because of the steady-state assumption. Let us assume that the electric mobility of positive ions, $\mu$, is constant irrespective of $x$. (This assumption requires that the species of ions does not change throughout $x$ and that the strength of the electric field is not very high.) The velocity of ions, $v$, is written as $v=\mu E$, where $E$ is the strength of the electric field. It is assumed that the positive or negative signs of $\mu$ depend on the positive and negative signs of $x$, respectively. The cases of $x>0$ and $x<$ 0 are expressed by the subscripts $\mathrm{A}$ and $\mathrm{C}$, respectively. The positive and negative signs of the parameters in this definition are presented in Table 1. The subscripts $\mathrm{A}$ and $\mathrm{C}$ are omitted when the formula is consistent, irrespective of $x>0$ and $x<0$.

Using the number density of charge particles, $n_{e}$, and electric charge $e$, the electric current density $j=e n_{e} v$ can be written as

$$
j=e n_{e} \mu E
$$

Ions in unit volume give momentum to the neutral gas by colliding with molecules. As a result, the neutral gas is given the electric body force $F_{e}$, which is macroscopically apparent. 


$$
F_{e}=\left(e n_{e}\right)_{+} E+\left(e n_{e}\right)_{-} E=\frac{j}{\mu_{+}}+\frac{j}{\mu_{-}}
$$

The subscripts + and - denote the force induced by positive charge and negative charge, respectively. The second equality comes from Eq. (1). Former studies reported that most of the carriers of negative electric charge are electrons around a flame. The electric mobility of electrons is much larger than that of ions (the mobility of ions is on the order of $10^{-4} \mathrm{~m}^{2} / \mathrm{sV}$, whereas the mobility of electrons is on the order of $\left.1 \mathrm{~m}^{2} / \mathrm{sV}\right)$. The term related to the negative charge carrier around the flame can be ignored in Eq. (2). In the area far from the flame, negative ions form through the electron attachment process and the mobility of the negative charge is not always much larger than that of ions.

Next, from Gauss's Law,

$$
\frac{d E}{d x}=\frac{e n_{e}}{\varepsilon}
$$

where $\varepsilon$ is the permittivity of air. After the elimination of $e n_{e}$ from Eqs. (1) and (3), under the assumption that $j / \mu$ is constant irrespective of $x$, the integration in $[0, x]$ yields

$$
\frac{1}{2} \varepsilon E^{2}-\frac{1}{2} \varepsilon E_{0}{ }^{2}=\frac{j}{\mu} x
$$

Here, $E_{0}$ is the strength of the electric field at $x=0$. After solving Eq. (4) for $E$, under the consideration that the potential is highest at $x=L / 2$, the integration in $[0, x]$ yields the electric potential $\varphi$

$$
\varphi=\frac{2 \sqrt{2}}{3 \sqrt{\varepsilon}} \sqrt{\frac{j}{\mu} x^{3}}+E_{0} x+\varphi_{0}
$$

Let us assume $E_{0}=0$ because the number density of the electric charge at the flame is high enough to counter the external electric field ${ }^{(1-4)}$. In Eq. (5), the value of parameter $\mu$ depends on the sign of $x$. By using the subscripts $\mathrm{C}$ and A for the $x<0$ and $x>0$ regions, the applied voltage $V$ between $x=-L / 2$ and $x=L / 2$ is written as

$$
V=\frac{1}{3 \sqrt{\varepsilon}}\left(\sqrt{-\frac{j}{\mu_{C}}}+\sqrt{\frac{j}{\mu_{A}}}\right) L^{\frac{3}{2}}
$$

From Eq. (2) and the discussion above, the electric body force on the flame is $j / \mu_{+}$. By solving Eq. (6) for $j / \mu_{\mathrm{C}}$, we get

$$
\frac{j}{\mu_{C}}=-\frac{\varepsilon V^{2}}{L^{3}} Q_{C}{ }^{2}
$$

Here, the coefficient $Q_{C}$ is

$$
Q_{C}=\frac{3}{1+\sqrt{\mu_{C} /\left(-\mu_{A}\right)}}
$$

The negative sign of the right-hand side of Eq. (7) indicates that the direction of the electric body force induced by the positive ions is downward. Because the unit of terms in Eq. (7) is $\mathrm{N} / \mathrm{m}^{3}$, both $j / \mu$ and $\varepsilon V^{2} / L^{3}$ show the electric body force (or pressure gradient). Eq. (7) indicates that the electric body force on the flame is proportional to $V^{2} / L^{3}$ and not $V / L$ $\left(=E_{\mathrm{eq}}\right)$. In addition, although the local strength of the electric field is a function of position $x$ (from Eq. (4)), the electric body force $j / \mu$ is constant irrespective of the position if the positive or negative sign of $x$ is decided.

\subsection{Estimation of parameters}

In this section, the orders of some parameters are estimated for subsequent discussion. First, let us consider the value of $Q_{C}$ in Eq. (8). Negative ions and electrons are considered to be carriers of negative charge, whereas positive ions are considered to be carriers of positive charge. If both are ions, $\mu_{C} \sim-\mu_{A}$, and $\mu_{C}<<-\mu_{A}$ when all negative charge carriers are electrons. This implies that the minimum value of $Q_{C}$ in Eq. (8) is $Q_{C}=3 / 2$ when $\mu_{C} \sim \mu_{A}$ and the maximum value is $Q_{C}=3$ when $\mu_{\mathrm{C}}<<-\mu_{\mathrm{A}}$. If Eq. (6) is solved for $j /\left(-\mu_{A}\right)$ and $Q_{A}$ is defined in the same way, the maximum $Q_{A}$ is $3 / 2$ when $\mu_{C} \sim-\mu_{A}$, and the 
minimum is $Q_{A} \sim 0$ when $\mu_{\mathrm{C}}<<-\mu_{\mathrm{A}}$.

Next, the relation between the electric field $E$ and the equivalent strength of the electric field, $E_{e q}=V / L$, is discussed. From Eq. (4) with $E_{0}=0$ and Eq. (7), $E$ is written as follows:

$$
E=-E_{e q} Q_{C} \sqrt{\frac{-x}{L / 2}}
$$

From Eq. (9), the maximum strength of the electric field, $E_{\max }$, is $Q_{C} E_{\text {eq }}$ at $x=-L / 2$. Because the maximum value of $Q_{C}$ is 3 , the strength of the electric field near the electrode is three times $E_{e q}$. In contrast, the position where $E_{\max }=-E_{e q}$ is $x /(L / 2)=0.11$ when $Q_{C}=3$, which implies that the strength of the electric field around the flame is not weak, at least on the cathode side, even in consideration of the change in the electric field.

Next, the validation of the model described in section 3.1 is discussed. Although the electric mobility of the ions is assumed to be constant irrespective of the strength of the electric field, the assumption necessitates the condition in which the mean free path of the ions is decided by their thermal velocity, which corresponds to $k T_{i}>>e E \lambda_{i n}$; Here, $k$ is the Boltzmann constant, $\lambda_{i n}$ is the mean free path of the ions, and $T_{i}$ is the ion temperature. $k T_{i} / e \lambda_{\text {in }}$ is around $4 \mathrm{kV} / \mathrm{cm}$. This means that the effectiveness of this condition is not apparent in the case of large electric field strength in our experiments.

In order to confirm the accuracy of the 1D assumption, the ratio between ion diffusion and ion movement velocity in the direction of the electric field, $\mu E_{e q} /\left(D_{i} / L\right)$, is estimated. The representative strength of the electric field $E_{e q}$ is around $4000 \mathrm{~V} / 50 \mathrm{~mm}$ and $D_{i}$ is $10^{-3}$ $\mathrm{m}^{2} / \mathrm{s}$ at maximum. If we use these values, $\mu E_{e q} /\left(D_{i} / L\right)$ becomes of the order of $10^{2}$, which indicates that it is possible to assume that the electric field distribution is one dimensional.

Because this study focuses on droplet combustion and the combustion time here is around $1 \mathrm{~s}$, the unsteady period is estimated because of the accuracy of the steady-state condition. The time when the electric field attains steady state can be estimated from the time when the ions arrive at the electrodes, $L / \mu E_{e q}$. Under the same electric field as that mentioned above, $L / \mu E_{e q}=2.5 \times 10^{-3} \mathrm{~s}$; this is much smaller than the combustion time. In addition, the unsteady time for gas convection is estimated from the characteristic time of natural convection, $((L / g) /(\Delta T / T))^{0.5}$. If $\Delta T / T=0.1$, this characteristic time is around $0.2 \mathrm{~s}$. This means that combustion of the gas phase can be assumed to be steady state, except for the initial phase of combustion. Although the flame radius and mass flux of fuel depend on time owing to combustion of the droplet, the electric field and flow field outside the flame can be considered to be steady state for a certain time period.

\section{Experimental Results and Discussion}

\subsection{Measurement of electric current density and observation of flame deformation}

In this study, flame deformation is observed and the electric current between the electrodes is measured during the combustion period.

Figure 2 shows the relationship between the applied voltage and the average electric current between electrodes during combustion for various electrode distances. It reveals that the electric current increases with an increase in applied voltage. This indicates that it is a "subsaturation" region of the voltage-current characteristic ${ }^{(2)}$ and it validates the assumption of $E_{0}=0$. However, for higher applied voltage, e.g., $L=50 \mathrm{~mm}$ and $V=10 \mathrm{kV}$, the electric current does not increase monotonically.

Next, the effect of flame deformation is described. Figure 3 shows typical photographs of flames in a vertical electric field. In all photographs, the electric ground is at the bottom side of the flame. This figure illustrates that the flame deforms toward the cathode (downward) with the application of electric voltage. Flame deformation is caused by the electric body force and it counters the buoyancy. To clarify the flame deformation, let us introduce the flame radii depicted in Fig. 3. The flame radius from the center of the droplet 
to the top edge of the flame is defined as $R_{1}$ and that from the center to the bottom edge is $R_{2}$. Figure 4 shows the degree of flame deformation $\left(R_{1} /\left(R_{1}+R_{2}\right)\right)_{\text {inst. }}$ plotted as a function of total flame length $R_{1}+R_{2}$. In this figure, the flame length is shown from its maximum point to its extinction point. The time intervals between the plots are around $0.35 \mathrm{~s}$ and the flame length decreases with time. This figure obviously shows that the flame deforms upward as a result of natural buoyancy in the absence of the electric field $(0 \mathrm{kV})$ and that it deforms downward in the presence of the electric field as a result of its electric body force with the increase in applied voltage. The flame becomes vertically symmetrical at around $V=2 \mathrm{kV}$, which corresponds to $\left(R_{1} /\left(R_{1}+R_{2}\right)\right)_{\text {inst. }}=0.5$; it deforms downward once more at $V=5 \mathrm{kV}$. Although the degree of deformation, $\left(R_{1} /\left(R_{1}+R_{2}\right)\right)_{\text {inst., }}$, varies with time because of the unsteady combustion of the droplet, the arithmetic average of the degree of deformation is defined as the deformation factor at a certain electrode distance and a certain applied voltage. For the average process, the value immediately before extinction is eliminated because the flame shape becomes vertically symmetrical before extinction. The instantaneous degrees of deformation range mostly between $93 \%$ and $110 \%$ of the averaged value. Figure 5 shows the flame deformation factor plotted as a function of applied voltage for various electrode distances. This figure reveals that the deformation factor principally decreases with increasing applied voltage and decreasing electrode distance. However, it is observed that at high voltages, the flame deforms upward once more.

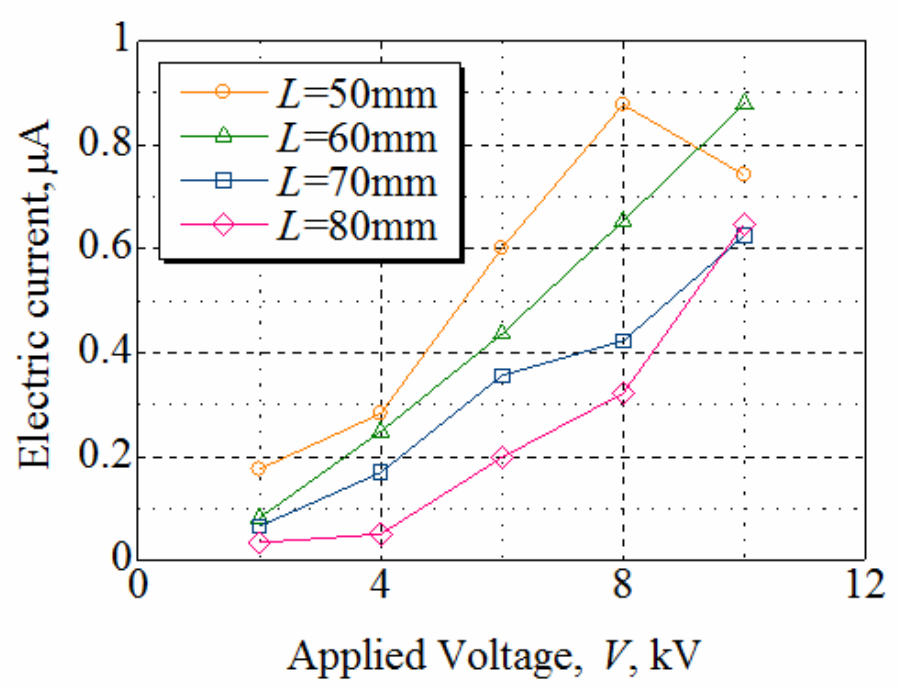

Fig. 2 Relationship between applied voltage and electric current
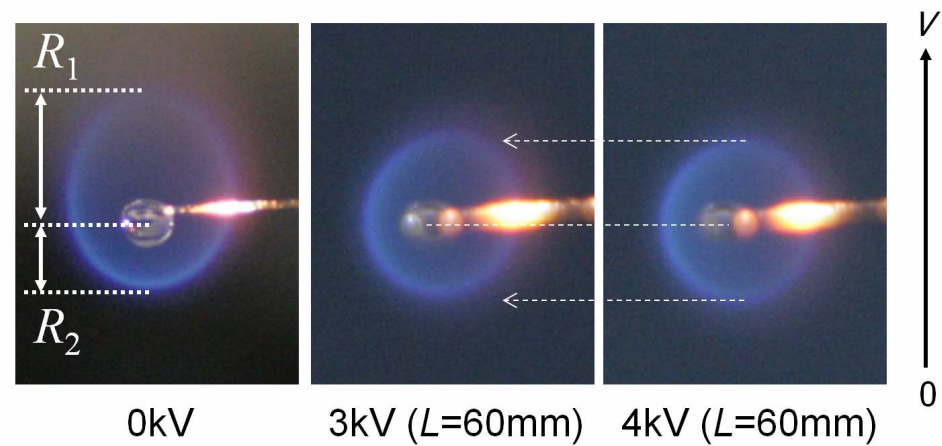

Fig. 3 Typical direct photographs of flame deformation 


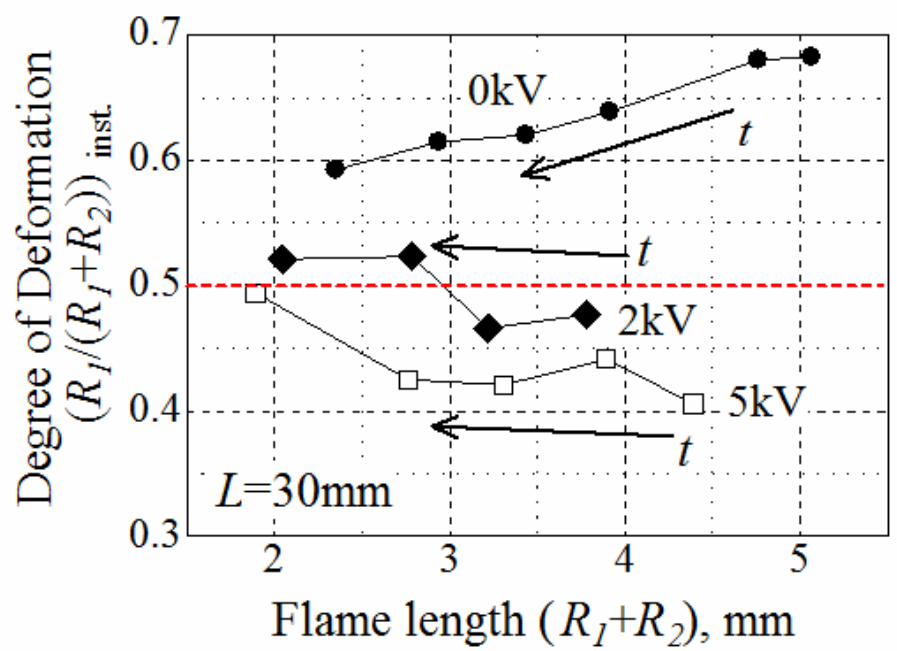

Fig. 4 Change in flame shape with time

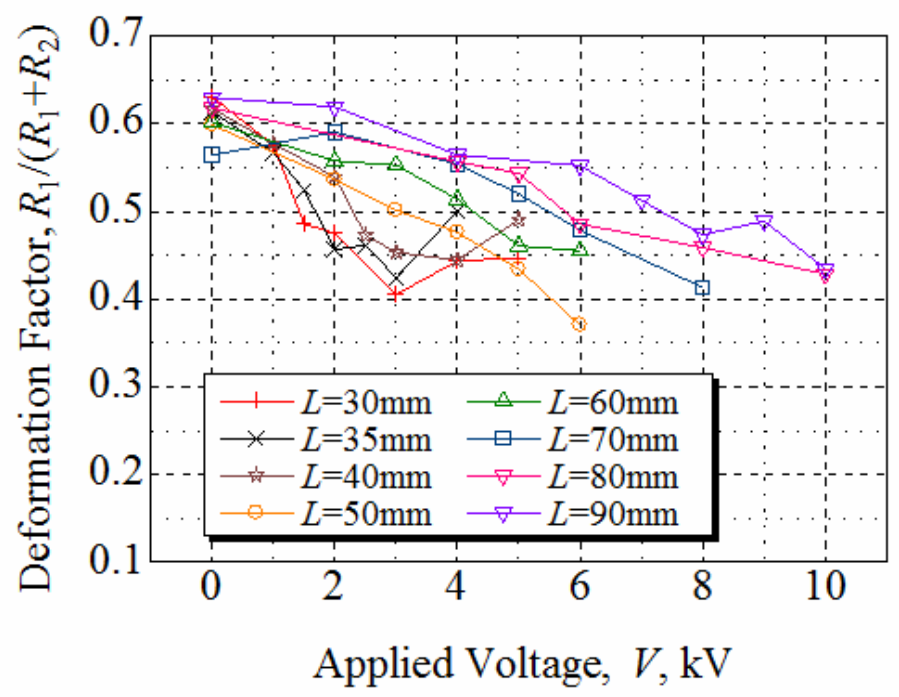

Fig. 5 Relationship between flame deformation factor and applied voltage

\subsection{Case of flame becoming vertically symmetrical}

Next, we focus on electric voltages in the case that the flame becomes vertically symmetrical. The electric voltage $V_{s y m}$ at which the flame is vertically symmetrical $\left(R_{1} /\left(R_{1}+\right.\right.$ $\left.R_{2}\right)=0.5$ ) is estimated from Fig. 5 by linear interpolation. The voltage $V_{s y m}$ is plotted as a function of electrode distance in Fig. 6. Because both axes are on a logarithmic scale, the slope in the figure corresponds to $n / \mathrm{m}$ if the flame deformation factor is a function of $V_{s y m}{ }^{m} / L^{n}$. From this figure, $n / m=1.57$ is obtained from a least-squares approximation. The flame deformation is induced by the electric body force, which is expected to be proportional to $V^{2} / L^{3}$ in Eq. (7) in section 3.1. If the buoyancy on the flame does not depend on electric voltage and electrode distance, the flame deformation can be described as a function of $V^{2} / L^{3}$. This indicates that the model described in section 3.1 explains this experimental result well. 


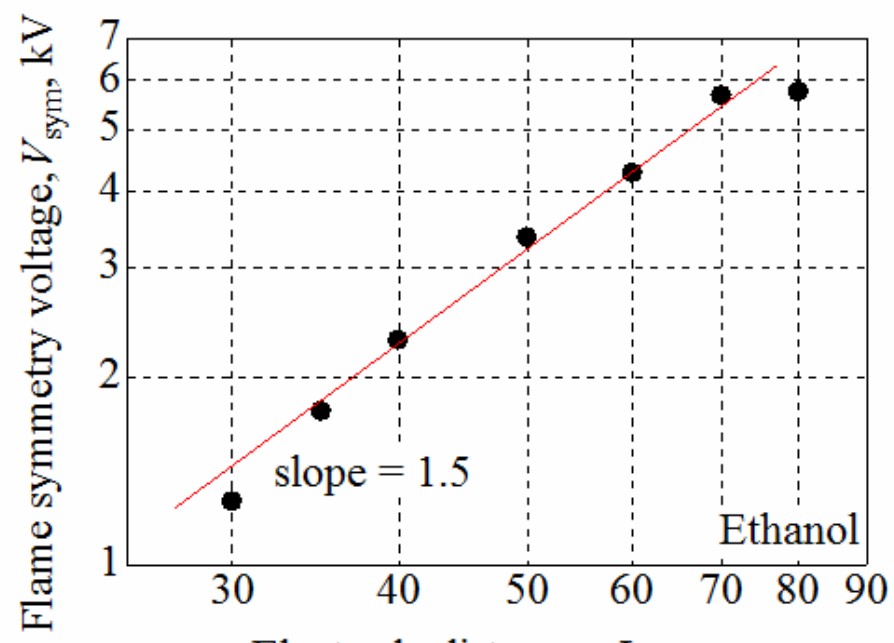

Electrode distance, $L, \mathrm{~mm}$

Fig. 6 Plot of voltages at which the flame becomes vertically symmetrical versus electrode distance

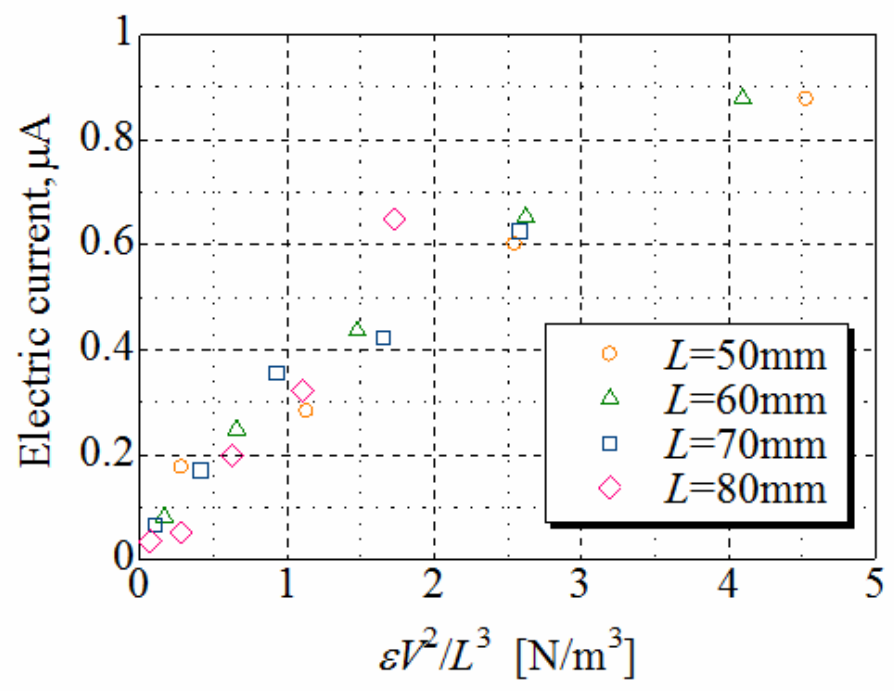

Fig. 7 Relationship between electric body force parameter and measured electric current

\subsection{Evaluation using electric body force}

From the discussion in section 4.2, it appears to be effective to rearrange the experimental results using $V^{2} / L^{3}$. This section extends the discussion to nonsymmetrical flame cases. Figure 7 shows electric current plotted as a function of representative electric body force $\varepsilon V^{2} / L^{3}$. For relatively low $\varepsilon V^{2} / L^{3}$, the plots align with a linear relation in spite of the electrode distance. The area where the flame becomes vertically symmetrical is less than $1 \mathrm{~N} / \mathrm{m}^{3}$. In the higher-force region $\left(\varepsilon V^{2} / L^{3}>2 \mathrm{~N} / \mathrm{m}^{3}\right)$, the tendency is different. If we assume $\varepsilon V^{2} / L^{3}=2 \mathrm{~N} / \mathrm{m}^{3}, E_{e q} \equiv V / L$ becomes around $1 \mathrm{kV} / \mathrm{cm}$ when $L=50 \mathrm{~mm}$. As discussed in session 3.2, the adopted model requires an electric field strength that is much less than 4 $\mathrm{kV} / \mathrm{cm}$. This indicates that the other model should be introduced for the higher-force region because the assumption $k T_{i}>>E \lambda_{\text {in }}$ is not effective any more. For the linear region $\varepsilon V^{2} / L^{3}<$ 
$2 \mathrm{~N} / \mathrm{m}^{3}$ in Fig. 7, the slope corresponds to $\mu_{C} A Q_{C}{ }^{2}$ from Eq. (7) and the value of slope is around $0.25 \times 10^{-6}\left[\mathrm{~m}^{4} / \mathrm{Vs}\right]$, where $A$ is the area of electric current $(j=i / A)$. If we assume $\mu_{\mathrm{C}}$ $=2.5 \times 10^{-4} \mathrm{~m} / \mathrm{sV}, Q_{C}=3$, and $A=0.01^{2} \mathrm{~m}^{2}$ which is estimated from the temperature distribution around the flame, then $\mu_{C} A Q_{C}{ }^{2}=0.23 \times 10^{-6}\left[\mathrm{~m}^{4} / \mathrm{Vs}\right]$; this agrees with the model result, considering the accuracy of the model and experiments.

Figure 8 shows the flame deformation factor considered in Fig. 5 rearranged using $\varepsilon V^{2} / L^{3}$. Figure 9 also shows the flame deformation factor rearranged using $E_{e q}=V / L$ instead of $V^{2} / L^{3}$. A comparison of these figures leads to the conclusion that flame deformation is caused by the electric body force and that it is better to understand the phenomena using the electric body force parameter $\varepsilon V^{2} / L^{3}$ than using the equivalent strength of the electric field, $V / L$. This suggests that the change in the electric field due to the existence of a flame should be considered when discussing the effect of an external electric field on combustion behavior.

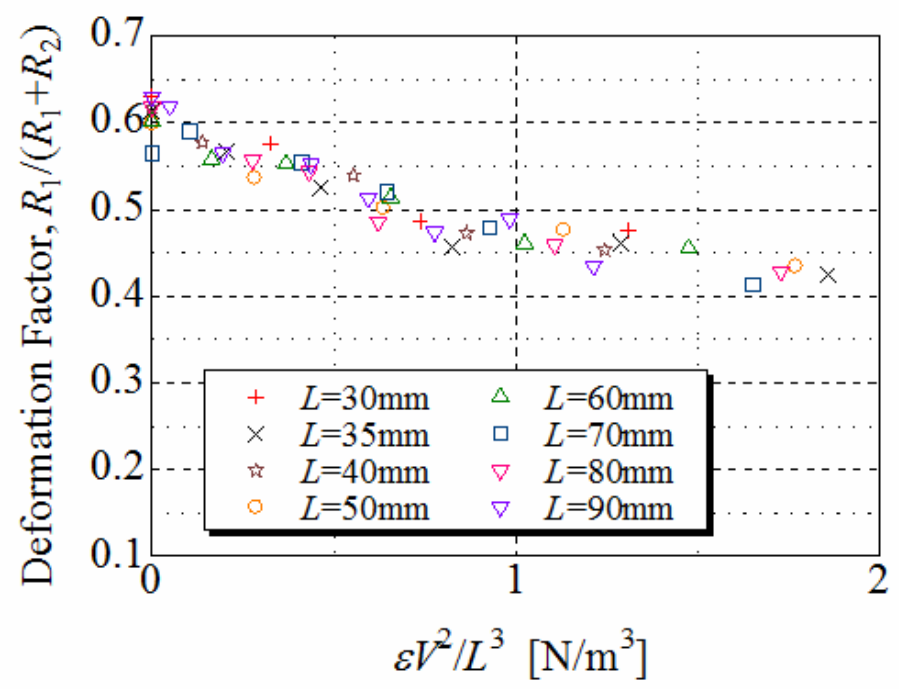

Fig. 8 Flame deformation factor plotted as a function of electric body force parameter

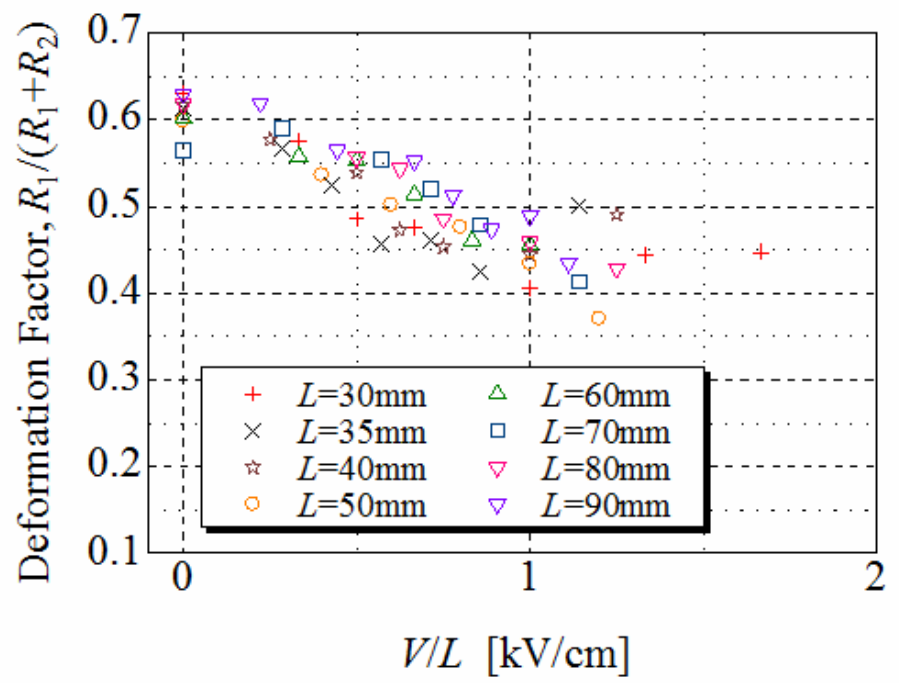

Fig. 9 Flame deformation factor plotted as a function of equivalent electric field 


\section{Summary}

Combustion experiments of ethanol droplets in a vertical electric field were performed with an apparatus having variable-distance electrodes, in order to discuss the change in the external electric field caused by the existence of a flame. A droplet was burned between the two horizontal parallel electrodes. Flame deformation and electric current were measured at various electrode distances and applied voltages. The applied vertical electric field generates electric body force in the downward direction and it counters the natural buoyancy, which makes the flame deform downwards. The relationship between the applied voltage $V$ and the electrode distance $L$ was investigated in the case that the flame became vertically symmetrical. The experimental results indicate that flame deformation is a function of $V / L^{1.5}$ and not $V / L$. This result agreed with the 1D steady-state analysis. On the basis of these results, the electric current was rearranged using the representative electric body force $\varepsilon V^{2} / L^{3}$, which agrees quantitatively with the $1 \mathrm{D}$ analysis. It can therefore be concluded that the effect of the electric field should be discussed using a parameter related to the body force $\varepsilon V^{2} / L^{3}$, not the overall equivalent strength of the electric field, $V / L$. These results indicate that the change in the electric field due to the existence of a flame should be considered when discussing the effect of an external electric field on combustion behavior.

\section{Acknowledgment}

The authors are grateful to Mr. M. Utsumi and Mr. A. Hiramoto for their assistance during the experiments and to Mr. G. Ianus for the editing support.

\section{References}

(1) Lawton, J., Mayo, P. J., and Weinberg, F. J., Electrical Control of Gas Flows in Combustion, Proceeding of the Royal Society of London, Series A., Vol. 303, (1968), pp.275-298.

(2) Lawton, J., and Weinberg, F. J., Electrical Aspects of Combustion, 1969, Clarendon Press, Oxford.

(3) Bradley, D., The Effects of Electric Fields on Combustion Process in Advanced Combustion Methods, 1986, Academic Press, pp.331-394.

(4) Carleton, F. B., and Weinberg, F. J., Electric Field-induced Flame Convection in the Absence of Gravity, Nature, Vol. 330, (1987), pp.635-636.

(5) Imamura, O., Kubo, Y., Osaka, J., Sato, J., Tsue, M., and Kono, M., A Study on Single Fuel Droplets Combustion in Vertical Direct Current Electric Fields, Proceedings of the Combustion Institute, Vol. 30, No. 2, (2005), pp.1949-1956.

(6) Carleton, F., Dunn-Rankin, D., and Weinberg, F. J., The Optics of Small Diffusion Flames in Microgravity, Proceedings of the Combustion Institute, Vol. 27, No. 2, (1998), pp.2567-2572.

(7) Papac, M. J., Dunn-Rankin, D., Stipe, C. B., and Lucas, D., N2 CARS Thermometry and O2 LIF Concentration Measurements in a Flame under Electrically Induced Microbuoyancy, Combustion and Flame, Vol. 133, No. 3, (2003), pp.241-254.

(8) Papac, M. J., and Dunn-Rankin, D., Modelling Electric Field Driven Convection in Small Combustion Plasma and Surrounding Gases, Combustion Theory and Modelling, Vol. 12, No. 1, (2008), pp.23-44. 